\title{
Judgement bias in goats (Capra hircus): Investigating the effects of human grooming
}

\author{
Luigi Baciadonna ${ }^{\text {Corresp.. }}{ }^{1}$, Christian Nawroth ${ }^{1}$, Alan G McElligott ${ }^{1}$ \\ ${ }^{1}$ Queen Mary University of London, Biological and Experimental Psychology, School of Biological and Chemical Sciences, London, UK \\ Corresponding Author: Luigi Baciadonna \\ Email address: luigi.baciadonna@gmail.com
}

Animal emotional states can be investigated by evaluating their impact on cognitive processes. In this study, we used a judgement bias paradigm to determine if short-term positive human-animal interaction (grooming) induced a positive affective state in goats. We tested two groups of goats and trained them to discriminate between a rewarded and a non-rewarded location over nine training days. During training, the experimental group $(n=9)$ was gently groomed by brushing their heads and backs for five min over 11 days (nine training days, plus two testing days, total time $55 \mathrm{~min}$ ). During training, the control group ( $\mathrm{n}=10$ ) did not experience any direct interaction with the experimenter, but was kept unconstrained next to him for the same period of time. After successful completion of the training, the responses (latency time) of the two groups to reach ambiguous locations situated between the two reference locations (i.e. rewarded/non-rewarded) were compared over two days of testing. There was not a positive bias effect after the animals had been groomed. In a second experiment, 10 goats were tested to investigate whether grooming induced changes in physiological activation (i.e. heart rate and heart rate variability). Heart rate increased when goats were groomed compared to the baseline condition, when the same goats did not receive any contact with the experimenter. Also, subjects did not move away from the experimenter, suggesting that the grooming was positively accepted. The very good care and the regular positive contacts that goats received from humans at the study site could potentially account for the results obtained. Good husbandry outcomes are influenced by animals' perception of the events and this is based on current circumstances, past experiences and individual variables. Taking into account animals' individual characteristics and identifying effective strategies to induce positive emotions could increase the understanding and reliability of using cognitive biases paradigms to investigate and promote animal welfare. 
1 Judgement bias in goats (Capra hircus): Investigating the effects of human

2 grooming

3

4 Luigi Baciadonna, Christian Nawroth and Alan G. McElligott

6 Queen Mary University of London, Biological and Experimental Psychology, School of

7 Biological and Chemical Sciences, Mile End Road, London E1 4NS, UK

9 Corresponding authors:

10 Luigi Baciadonna

11 Email: Luigi.baciadonna@gmail.com

13 Alan G. McElligott

14 Email: a.g.mcelligott@qmul.ac.uk; Tel: + 44 (0)20 78826682.

15

16 Short title: Judgement bias in goats 


\section{Abstract}

Animal emotional states can be investigated by evaluating their impact on cognitive processes. In this study, we used a judgement bias paradigm to determine if short-term positive human-animal interaction (grooming) induced a positive affective state in goats. We tested two groups of goats and trained them to discriminate between a rewarded and a non-rewarded location over nine training days. During training, the experimental group $(n=9)$ was gently groomed by brushing their heads and backs for five min over 11 days (nine training days, plus two testing days, total time $55 \mathrm{~min}$ ). During training, the control group $(n=10)$ did not experience any direct interaction with the experimenter, but was kept unconstrained next to him for the same period of time. After successful completion of the training, the responses (latency time) of the two groups to reach ambiguous locations situated between the two reference locations (i.e. rewarded/non-rewarded) were compared over two days of testing. There was not a positive bias effect after the animals had been groomed. In a second experiment, 10 goats were tested to investigate whether grooming induced changes in physiological activation (i.e. heart rate and heart rate variability). Heart rate increased when goats were groomed compared to the baseline condition, when the same goats did not receive any contact with the experimenter. Also, subjects did not move away from the experimenter, suggesting that the grooming was positively accepted. The very good care and the regular positive contacts that goats received from humans at the study site could potentially account for the results obtained. Good husbandry outcomes are influenced by animals' perception of the events and this is based on current circumstances, past experiences and individual variables. Taking into account animals' 
40 individual characteristics and identifying effective strategies to induce positive emotions

41 could increase the understanding and reliability of using cognitive biases paradigms to

42 investigate and promote animal welfare.

43 
44

45

46

47

\section{Introduction}

The assumption that animals are sentient and therefore able to experience emotions creates the new challenge of assessing their emotions and, when possible, to identify strategies to promote positive emotional experiences $[1,2]$. The assessment of animal emotions is difficult, because they cannot report their emotional experiences through language [3-5]. The use of a multicomponent approach in which several parameters (e.g. behaviour and neurophysiology) are taken into account allows emotions to be assessed indirectly $[5,6]$.

The use of the judgement bias in animals has been inspired by studies carried out in humans, because the ways that people perceive, interpret and judge information is influenced by their emotions and feelings [3,7]. People with depression or anxiety are more likely to perceive social information as threatening and pay more attention to negative stimuli [8]. In animals, the impact of emotional states on behavioural expression has been identified through behavioural and physiological changes induced by specific environmental stimuli. For example, unfamiliar and unexpected objects generate a startle response in sheep (Ovis aries) [6]. Furthermore, when expectations about food were violated, lambs increased locomotor activity and there was a decrease in the parasympathetic influence on their cardiac activity [9].

Recently, the cognitive bias paradigm has been used to examine the interactions between emotional states and cognitive processes (e.g. attention, judgment and memory) in animals $[3,10,11]$. The assumption underlying this paradigm is that an experimentally induced alteration of an emotional state generates a behavioural response bias (e.g. judgement) that is linked with the emotional experience of the 
67 subject. Thus, the evaluation of ambiguous stimuli (i.e. novel stimuli introduced in

68

69 between previously learned positive and negative cues) is affected by the emotional states experienced. There is strong evidence that the induction of negative emotional states generates a negative judgement of ambiguous stimuli $[3,10]$. For example, livestock exposed to long-term stressors [12], psychological stress [13] or pharmacological treatments [14] show negative judgement biases. By contrast, the study of positive judgement biases has produced inconsistent findings. A positive judgement bias is associated with short-term (i.e. a few days or weeks) changes to housing conditions[15] (although not always confirmed; [16]), long-term good care [17], with pharmacological treatment using diazepam, morphine [14], and specific manipulations (i.e. tickling) [18]. Rats (Rattus norvegicus) treated with oxytocin did not display a shorter latency to approach ambiguous trials compared to rats treated with saline solution. However rats (regardless of treatment), were significantly slower on the aversive trials compared to the ambiguous trials, and thereby indicating an overall positive bias [19]. Contrary to the predictions, positive judgement biases have also been found when animals are released from short-term stressors [20]. These inconsistencies could be attributed to the poor assessment of the rewarding or non-rewarding (punishment) properties of the stimuli adopted or due to a lack of knowledge of animals' cognitive abilities to quantify and discriminate the ambiguous stimuli [10]. Interactions between humans and animals offer an interesting and valid way for testing the effects of induced positive emotions on judgement bias in animals. The quality of the relationship between human handlers and farm livestock has a large effect on animal wellbeing, productivity, and success in handling animals easily [21,22]. For 
90

91

92

93

94

95

96

97

98

99

100

101

102

103

104

105

106

107

108

109

110

111

112

example, regular positive contact between humans and animals reduces fear reactions

in animals [22]. Similarly, positive contact between humans and animals (e.g.

petting/grooming) can generate an affinity for the stockperson, with increased

motivation to search and approach the caretaker [23] and calming effects $[21,24]$.

Farm livestock might be particularly sensitive and responsive to positive

interactions with humans due to their long history of domestication $[25,26]$. In cattle (Bos

taurus), grooming was associated with a reduction in cortisol levels, changes in cardiac

activity linked with specific body parts $[22,27]$ and also with changes in ear postures

[28]. In lambs, gentle tactile contact with humans after a period of chronic stress is

associated with a positive judgement bias [29]. With the aim of further exploring the use

of specific human-animal interactions to induce positive emotional states in farm

livestock, we investigated whether short-term strategies to boost the effects of routine

positive care in goats would induce a positive judgement bias ([17]; Experiment 1). We

predicted that grooming would induce a positive state and in turn optimistic-like bias

during a judgement bias test. The second aim of the study was to measure the

physiological changes (i.e. heart rate and heart rate variability) and the behaviour

associated with grooming (i.e. proximity to the experimenter), to determine whether this

procedure was effective in inducing emotional changes (valence and arousal;

Experiment 2)

2. Methods

2.1. Experiment 1: Judgement bias

2.1.2. Subjects and management conditions 
The study was carried out at a goat sanctuary (Buttercups Sanctuary for Goats,

114 http://www.buttercups.org.uk; Kent, UK). Nineteen adult goats (10 females and nine

115

116

117

118

119

120

121

122

123

124

125

castrated males) of various breeds and age (Table 1) were tested from April to May 2014. Subjects were allocated either to an "experimental group" ( $n=$ nine goats, five females and four castrated males) or to a "control group" ( $n=10$ goats, five females and five castrated males). Goats that were used in this study had been at the sanctuary for a minimum of one year (range: 1 to 14 years). Employees and sanctuary volunteers provided routine care for the animals and therefore the goats were fully habituated to human presence and handling $[17,30]$. During the day, all goats were released together into one or two large fields that also provide shelters. During the night, they were kept indoors in individual or shared pens (average size $=3.5 \mathrm{~m}^{2}$ ) with straw bedding, within a large stable complex. Goats had ad libitum access to hay, grass (during the day) and water and were also fed with a commercial concentrate in quantities that varied according to their health and age.

\subsubsection{Treatment}

Goats of the experimental group were gently groomed by one of the authors (LB) with a commercial animal brush. LB has been involved in research at the study site since 2011 and was therefore very familiar to the animals. Goats were familiar with the brush because this was occasionally and intermittently used by staff and volunteers at the sanctuary to remove dirt from their hair, and not for inducing positive emotional states per se. Animals were groomed on the frontal and lateral part of the head and behind the horns and on the back (close to the base of the tail). These body parts were 
136 selected because animals at the sanctuary often scratch these same areas against

137 trees branches or large boulders (LB, personal observation). The experimental group

138 received five min of grooming before the training session, for nine days, over two

139 weeks. They also received five min of grooming with the experimenter before the test

140 session, for two days. Therefore, in total, each animal received 55 min of grooming over

14111 days. We expected grooming to induce a positive emotional state [27-29,31]. The

142 control animals were kept unconstrained adjacent to the experimenter for the same

143 period of time as the goats in the experimental group (five min for nine days of training,

144 plus two days of testing), but were not groomed.

\subsubsection{Experimental apparatus}

An experimental apparatus $(5 \mathrm{~m} \times 6.25 \mathrm{~m})$ was set up and placed in one of the

fields that is part of the goats' normal daytime range [20]; Figure 1). It consisted of a start pen $(5 \mathrm{~m} \times 1.25 \mathrm{~m})$ connected by a door to a central arena and five corridors (corridor length $=2.50 \mathrm{~m}$, corridor width $=1.25 \mathrm{~m}$ ) made of sheeted livestock fencing

151 (height $=1 \mathrm{~m}$ ). The central arena allowed opening or closing of a manually operated gate to provide access to the corridors. The choice of a specific corridor (either on the right or left side of the arena) was rewarded with a mix (approximately $70-80 \mathrm{~g}$ ) of apples and carrots ("positive corridor"). The corridor at the opposite side of the arena was never rewarded ("negative corridor"). Three ambiguous corridors were positioned between the positive and negative corridors. One ambiguous corridor was positioned next to the positive corridor ("near positive"), one was positioned in the middle ("middle

158 corridor"), and one next to the negative corridor ("near negative"). The ambiguous 
159 corridors were never rewarded in order to avoid associations between these locations

160 and the presence of a food reward. A grey bucket with food (positive corridor) or an

161 empty grey bucket (negative or ambiguous corridors) was placed at the end of the

162 corridors. The buckets were covered with a plastic lid (8 $\mathrm{mm}$ thick) in order to reduce

163 olfactory cues that could have indicated the presence of food.

164

165

\subsubsection{Habituation}

To familiarise the animals with the experimental apparatus, each goat was

167 individually placed in the apparatus twice, for 12 min, over two consecutive days. Each session consisted of two min in the start pen, followed by 10 min of exploration inside the arena. During the habituation phase, all five corridors were opened and a small quantity of food (mix of apple and carrots) was scattered in the enclosure to encourage exploratory behaviour. A grey bucket with a lid was placed in the middle of the central arena. This bucket was used also during the training and test phases. This allowed the goats to associate the grey bucket with the food reward and to practice how to remove

174 the lid and retrieve the food.

\subsubsection{Judgement bias training}

Nine goats (five females and four males) were trained to expect food on the right side (positive corridor, four goats from the experimental group and five from the control

179 group) and 10 goats (five females and five males) were trained to expect food on the left side (positive corridor; five goats from the experimental group and five goats from the control group, Table 1). Goats were tested in random order. The experimental group 
182 (nine goats) was groomed for five min before starting the training procedure. The control 183 group (10 goats) was also placed in the starting pen for five min before the training and

184 kept adjacent to the experimenter. The ambiguous corridors (near negative, middle, and 185 near positive) remained closed during this phase. Only one corridor at a time (either 186 positive or negative) was open. During the first session of training, all goats received 187 two consecutive positive trials followed by two consecutive negative trials plus two 188 additional trials where they were trained to reach the positive and negative corridors 189 alternatively (six trials in total). This was, in order to facilitate discrimination between the 190 two locations. For the other training sessions, a pseudo-random order with no more 191 than two consecutive positive or negative trials and with the same number of positive 192 and negative trials per session was used [17]. A significant shorter latency to reach the 193 positive than the negative corridor was obtained for all goats on the second day of 194 training (linear mixed-effects models: $p \leq 0.001$ ). At the end of each training day, the 195 average latency time to reach the positive and negative corridors was calculated. The training ended after nine days, when the latency to approach the positive corridor was on average less than $5 \mathrm{~s}$ and the latency to reach the negative corridor was more than $100 \mathrm{~s}$.

\subsubsection{Judgement bias test}

The test phase was conducted over two consecutive days. During each testing day, goats were tested over seven trials (i.e. one session). In particular, they were tested three times with the ambiguous corridor, two times with the positive corridor, and two times with the negative corridor. The positive and negative trials were repeated 
205 twice, as a reminder. The ambiguous corridors were opened in random order and were

206 alternated with the positive and the negative corridors. Indeed, the ambiguous corridors

207 were tested after the positive or after the negative corridor over the two days [17].

208

209

\subsubsection{Training and testing procedure}

210 During the training and testing trials, the goats were individually brought to the start pen

211 and groomed (only the experimental group) for five min. After grooming, the

212 experimenter opened the gate of the appropriate corridor. The bucket was filled with

213 food for a positive trial or we pretended to fill the bucket (making noise with food) for a

214 negative or an ambiguous trial. The bucket was subsequently covered with the plastic

215 lid. Next, the start pen door was opened to allow the goat to enter the central arena. The

216 experimenter waited for the goat to reach and cross the line and allowing the time to eat

217 the food (positive corridor), or to reach and cross the line at the beginning of the corridor

218 before returning to the start pen. A short inter-trial interval ( $<1 \mathrm{~min})$ followed, to prepare

219 for the next trial. During each training and test session, the time from when the animal's

220 two front legs passed the line on the gate at the entrance of the central arena to the

221 time when they reached and crossed the line at the entrance of the target corridors with

222 the two front legs was recorded. If the goat did not enter the central corridor from the

223 start pen in $90 \mathrm{~s}$, the door was closed and training/testing session continued. If the goat

224 did not cross the line at the entrance of the open corridor, it was brought back to the

225 start pen after $180 \mathrm{~s}$ and the training/testing session continued to the next trial. All

226 sessions were recorded using a digital video camera placed behind the subject (Sony

227 HDR-CX190E). The experimenter (who was not blind to the treatment), recorded the 
228 latency time directly. A second observer, blind to the experimental hypotheses, scored

$22920 \%$ of the total sessions to test the reliability of the latency times recorded [32]. The

230 inter-observer agreement for latency time was high (Spearman rank correlation; $r_{\mathrm{s}}=$

$2310.976 ; p<0.001)$.

232

233

2.2 Experiment 2: Physiological effects of the grooming

234

235

236

237

238

239

240

241

242

243

244

245

246

247

248

249

250

\subsubsection{Subjects and management conditions}

The general management conditions of the animals are described in section

2.1.2. Ten goats (five females, five castrated male) were tested to assess the effect of grooming on the physiological level during December 2015. Only six goats used in Experiment 1 were available for Experiment 2 and therefore, four goats were naïve when they participated in Experiment 2. Goats were tested twice on two nonconsecutive days; once without being groomed with an experimenter close to the subject (control), and the second time they were actively groomed for five min by the experimenter. The aims of Experiment 2 were to assess the physiological changes of both branches of the autonomic nervous system (sympathetic and parasympathetic) using heart rate and to examine the activation of the parasympathetic system only using heart rate variability $[5,33]$. The behaviour associated with grooming (i.e. proximity to the experimenter) was also recorded. These two different types of data (i.e.

physiological and behavioural) allowed us to determine whether the grooming was effective in inducing emotional changes in valence and arousal

\subsubsection{Treatment and physiological recordings}


The goats were groomed with a commercial animal brush in one of the indoor

252 pens where they were normally kept overnight. Goats were groomed on the frontal and

253 lateral part of the head, the part behind the horns and on the back (close to the tail). The physiological parameters were recorded using a non-invasive device, fixed to a belt placed around the goat's chest (EC38 Type 3, BioHarness Physiology Monitoring System, Zephyr Technology Corporation, Annapolis, MD, U.S.A.). Heart rate was measured using the BioHarness system. The week before the test commenced, a small patch of hair $(7 \mathrm{~cm} \times 15 \mathrm{~cm})$ was clipped so that the heart rate monitor worked more effectively. The BioHarness was also attached to the animal for a short period of time (five min) in order for habituation to occur. The habituation was conducted for a short period of time because the goats that participated in this study had previously experienced wearing the device during other research $[5,34]$. The continuous ECG trace was transmitted online to a laptop (ASUS 200E) and stored using software (AcqKnowledge 4.4, BIOPAC System Inc) for later analyses. When the heartbeats were clearly visible on the ECG trace, 10 s sections ("start", "middle" and "end"; mean \pm SD for each of the three sections: "start": $10.12 \pm 0.68 \mathrm{~s}$; "middle": 10.00 $\pm 0.71 \mathrm{~s}$; "end": $10.06 \pm 0.74 \mathrm{~s}$ ) were selected and analysed. Heart rate and heart rate variability (i.e. root mean square of successive interbeat interval differences, RMSSD) were analysed from the ECG trace. To improve the quality of ECG trace, any electrical noise was removed by selecting Line frequency of $50 \mathrm{~Hz}$ (from AqcKnowledge>Transform>Digital Filters $>$ Comb Band Stop). Baseline drift and movements artefact were also removed using a high pass filter at fixed cut off frequency

273 of $1 \mathrm{~Hz}$ (from AqcKnowledge $>$ Transform $>$ Digital Filters $>$ IIR $>$ High Pass). The 
274 AcqKnowledge software provided the heart rate (beats/min) automatically. Individual

275 intervals between heartbeats were also extracted to calculate RMSSD. All sessions

276 were recorded using a digital video camera placed behind the subject (Sony HDR-

277 CX190E). The total time that the experimenter actively groomed the goats was recorded 278 during the grooming session.

\subsection{Data analysis}

For Experiment 1, the average latency to reach the positive and negative location on each training day was calculated for each subject. For the testing phase, the latency to reach the locations over the two sessions was averaged for each goat [17]. The latency data from the training and testing phases were analysed with linear mixedeffects models (Linear Mixed Effect Model (LMM); Imer function, Ime4 library; [35]) in R 3.2.2 [36]. The linear mixed-effects model analysis allowed us to examine the following variables: "treatment" (groomed vs. control), "location" (positive, negative, near positive, middle, and near negative), "age", "training day", and "side" (reward side) as fixed effects. The identity of the goats was included as a random factor to control for repeated measurements of the same subjects. The LMM allows the elimination of the nonsignificant variables considered in the model if does not cause any significant reduction in goodness of fit of the model, using a standard model simplification procedure. The two models with and without each term, both fitted with the maximum likelihood method (ML), were compared using a likelihood ratio test. The results are presented after model simplification and with restricted maximum likelihood method (REML). When an 296 interaction effect was found, further post-hoc comparisons were performed using LMM, 
297 including control factors that remained in the final models. Bonferroni correction was

298 applied to the post-hoc comparisons. Q-Q plots and scatterplots of the residuals of the

299 model were inspected visually. In order to meet the assumptions, the latency times were

300 transformed using a reciprocal transformation $\left(1 / X_{\mathrm{i}}\right)$.

301 The data for Experiment 2 were analysed using a LMM that allowed us to

302 investigate for effects of the following variables: "treatment" (groomed/control), "section"

303 (the part selected for the HR and HRV; Start, Middle and End) and "sex" as fixed

304 effects. The same standard elimination procedure used for the judgement bias

305 experiment was applied as previously described. The identity of the goats was included

306 as a random factor to control for repeated measurements of the same subjects. Q-Q

307 plots and scatterplots of the residuals of the model were inspected visually to verify the

308 test assumptions.

309

310

\section{Ethical Note}

Animal care and all experimental procedures were conducted in accordance with

312 the Association for the Study of Animal Behaviour (ASAB) guidelines [37]. The study was approved by the Animal Welfare and Ethical Review Board of Queen Mary

314 University of London (25042014FdQMUL). The tests were non-invasive and lasted less

315 than 10 min (including the preparation time for adjusting the belt around the chest of the

316 subject and the grooming treatment) for each animal. Behaviours indicating stress

317 (frequent vocalizations and rapid movements away from the experimenter) were

318 monitored throughout the exposure to grooming. None of the goats displayed

319 behavioural signs of stress during the experiment. 


\section{5. Results}

322

323

324

325

326

327

328

329

330

331

332

333

334

335

336

337

338

339

340

341

342

\subsubsection{Experiment 1: Judgement bias training}

An interaction effect between "training day" and "location" was found (LMM: $X^{2}{ }_{(1)}$ $=202.35, p<0.001)$. Post-hoc analyses, after Bonferroni correction $(p \leq 0.01)$, indicated that the goats learned the task on the second day of training $(p<0.001)$. Goats reached the positive corridor faster (latency mean $=15.60 \pm 4.96 \mathrm{~s}$ ) than the negative corridor (latency mean $=27.61 \pm 7.04 \mathrm{~s} ; \mathrm{n}=19$ goats, Figure 2). An interaction effect between "sex" and "location" was also found $\left(\mathrm{LMM}: X_{(1)}=6.97, p=0.008\right)$. Post-hoc analyses, after Bonferroni correction $(p \leq 0.01)$, revealed that females $(p<0.001)$ and males $(p<$ $0.001)$ approached only the non-rewarded corridor differently $\left(\mathrm{LMM}: X^{2}{ }_{(1)}=4.51, p=\right.$ 0.03 ; rewarded corridor LMM: $X^{2}{ }_{(1)}=0.64, p=0.42$. The difference on the non-rewarded corridor was not retained after correction $(p>0.01)$. The other terms included in the initial model, namely "treatment" $\left(\mathrm{LMM}: X^{2}{ }_{(1)}=0.21, p=0.64\right)$, "side" $\left(\mathrm{LMM}: X^{2}{ }_{(1)}=1.22\right.$, $p=0.26)$, "age" $\left({\operatorname{LMM}:{ }^{2}}_{(1)}=2.44, p=0.11\right)$, and the interaction terms $(p \geq 0.05)$, did not significantly affect the latencies time during the training phase.

\subsubsection{Experiment 1: Judgement bias test}

The model selection procedure for the testing sessions revealed an effect of location on the general latencies $\left(\mathrm{LMM} X^{2}{ }_{(1)}=89.55, p<0.001\right)$, with goats reaching the positive corridor faster than the negative one, and the ambiguous corridors with intermediate latencies (Figure 3). There was no interaction effect between the "treatment" and "location", on the latency to reach the five corridors $\left(\mathrm{LMM}: X^{2}{ }_{(4)}=4.10\right.$, 
$343 p=0.39$ ). A weak interaction effect between "treatment" and "sex" was found (LMM:

$\left.344 X^{2}{ }_{(1)}=3.63, p=0.056\right)$. Post-hoc analyses, after Bonferroni correction $(p \leq 0.01)$

345 revealed that females were slower than males overall $\left(\operatorname{LMM}: X^{2}{ }_{(1)}=6.29, p=0.01\right)$,

346 regardless of treatment condition. Males reached the five corridors faster (mean latency

$347=51.74 \pm 10.19 \mathrm{~s}$ ) than females (mean latency $=62.37 \pm 10.17 \mathrm{~s} ; \mathrm{n}=19$ goats). After

348 the Bonferroni correction, the effect of "treatment" was not retained $(p>0.05)$. An effect

349 of "age" on the latency to reach the locations was also found $\left(\mathrm{LMM}: X^{2}{ }_{(1)}=5.53, p=\right.$

350 0.01). Goats aged one to seven year old reached all corridors faster than those aged 8-

35114 years old (mean latency: $42.32 \pm 9.38 \mathrm{~s}, N$ goats $=8$; mean latency: $68.25 \pm 10.20$,

$352 N$ goats =11). To summarize, there was no effect of grooming on the approach latencies

353 to the five corridors. However, females were slower than males when approaching the

354 corridors. An effect of age was found, with younger subjects faster than older ones.

5.2. Experiment 2: Physiological activation during the grooming

An effect of "treatment" on heart rate was found $\left(\mathrm{LMM}: X^{2}{ }_{(1)}=11.63, p<0.001\right)$.

Heart rate was higher when the goats were groomed (mean BPM: $103.90 \pm 2.58$ )

compared to the control (close to the experimenter without being groomed; mean BPM:

$95.59 \pm 2.27$, Figure 4). The other terms included in the initial model, namely "sex"

$\left(\mathrm{LMM}: X_{(1)}=0.06, p=0.80\right)$, "section" $\left(\mathrm{LMM}: X^{2}{ }_{(2)}=1.47, p=0.47\right)$, and the interaction

terms $(p \geq 0.05)$, did not significantly affect heart rate. "Treatment" had no effect on

heart rate variability $\left(\mathrm{RMSSD}\right.$; LMM: $\left.X^{2}(1)=0.04, p=0.83\right)$. The other terms included in

$4.59, p=0.10)$, and the interaction effect $(p \geq 0.05)$, did not affect the heart rate 
366 variability. The videos showed that goats did not avoid being groomed (i.e. they did not

367 move away when the experimenter approached) for most of the time (mean: $287 \pm$

$36810.50 \mathrm{~s} ; 95.66 \%$ of the total amount of time allowed). Heart rates increased when the

369 goats were groomed compared to when they were kept inside the pen with the

370 experimenter without engaging in any contact (Figure 4).

371 
373

374

375

376

377

378

379

380

381

382

383

384

385

386

387

\section{Discussion}

Human-animal interactions can have huge impacts on the emotional lives of animals [22]. Investigating this topic can provide valuable information to promote positive emotions and psychological wellbeing in animals [7]. In this study, we used short-term positive human-animal interaction (i.e. grooming) to attempt to induce positive emotional states in goats $[24,31,38]$. We hypothesised that grooming would induce positive emotional states, which in turn would lead to an optimistic-like bias. We found no significant differences in the judgement of ambiguous stimuli between goats that had been groomed and goats that had not received this treatment. However, a significant effect of age on the latencies to reach the corridors was revealed, with younger goats faster than older goats in choosing a corridor. These findings may indicate that grooming did not induce strong enough positive emotional states in goats, or that the performance in the judgement bias test was not influenced by positive emotions. In the second experiment, we found that the heart rates of the goats increased as a result of being groomed, and also that the animals accepted the grooming most of the time. This suggests that the treatment was perceived by the animals, but could not be detected during the judgement bias test. The use of the judgement bias test in farm animals is controversial and has produced discordant findings $[11,15,25]$. More research is needed to identify effective strategies to induce positive emotions and to develop assessment tools able to detect emotional changes, especially positive ones $[6,7]$.

To test the effectiveness of the manipulation we performed an experiment in which physiological activation was recorded in two treatments (i.e. control, with no 
396 contact with the experimenter, and grooming). We found that heart rates were higher

397 during grooming compared to the control treatment. In combination with the behavioural

398 finding that animals accepted being groomed for most of the time $(95.66 \%)$ by the

399 experimenter, this suggests that the grooming not only induced an emotional change in

400 arousal, but also a change that was perceived as positive. This supports the hypothesis

401 that grooming was effective in inducing an emotional change, and that the judgement

402 bias task might have not been able to detect this change.

403

Heart rate and heart rate variability measurements are good indicators of

404

emotional arousal and valence when used in combination with other parameters, such

as behavioural responses and postures [5,39,40]. For example, sheep [41] exhibited a

higher inter-heartbeat interval ( $R-R$ interval) and higher heart rate variability when

groomed compared to when they were standing in their home pen or in isolation. Lambs

408

409

410

411

412

413

414

415

416

417

418

regularly stroked in early age and with strong bonds with humans also showed lower

$H R$ in the presence of their caregiver and while groomed, and higher RMSSD when

compared with lambs that were not stroked [24]. In cattle, stroking and gentle human

voices were associated with reduced heart rate following an aversive event (veterinary

procedure; [42]). However, in dogs, the RMSSD did not increase as expected whilst

experiencing a positive situation (palatable food) $[43,44]$. The activation of the vagal

tone, in dogs, has been suggested to occur when animals experience a further increase

in the positive emotion that they were already experiencing [43]. In our case, it is most

likely that goats had not experienced the grooming for a long enough period of time.

This might have prevented them from developing a specific bond with the experimenter and from showing changes in heart rate variability as a consequence. 
$42055 \mathrm{~min}$ in total over six weeks) may not have been strong enough to further improve and

421 boost the positive emotional states and experience of the goats that we used $[17,45]$.

422 Goats at our study site are kept in generally excellent conditions (i.e. according to the

423 DEFRA Codes of Recommendation for the Welfare of Goat; $[17,46])$ and they are used

424 to experience positive interactions with people. These conditions are not comparable to 425 those of laboratory animals or to the situations of chronic stress to which farm animals 426 are normally exposed before experiencing a positive event (e.g. gentle tactile contact 427 with a human) in a judgement bias test study [12]. In addition, although we selected the 428 body parts that were groomed because animals at the sanctuary often scratch 429 themselves against tree branches or large boulders (LB, personal observation), these 430 parts might have not been appropriate to respond to a gentle tactile stimulation [47]. 431 Previous research has indicated the importance of selecting specific body parts for the 432 grooming to be effective, such as regions touched during social behaviour $[27,28,31]$. 433 For example, cattle groomed on the ventral part of the neck showed less avoidance 434 behaviour compared with cattle groomed in the lateral side of the chest or withers [31]. 435 The efficacy of the grooming could be linked to the person who performed the 436 manipulation [27]. In order to generalise the results, it would useful to use more than a 437 single experimenter to perform the grooming We found that age affected the overall performance in the judgement bias test. 439 Younger animals approached the corridors faster than older ones (i.e. 1-7 year old 440 goats faster than those aged 8-14 years old). This effect of age was not found during 441 the training phase and suggests that age differences are unlikely to be related to 
442 physical effects. A faster approach during the judgement bias test could be due to

443 impulsivity, defined as incapability to refrain from a motor response [48]. Impulsivity has

444 been associated with young ages in humans and non-human animals $[49,50]$ and

445 declines gradually with increasing age $[51,52]$. Thus, age could affect the use of specific

446 coping strategies in unpredictable/new situations. To avoid any potential confounding

447 effect associated with the impulsivity and motivation the use of Go/Go or Active Choice

448 task has been suggested as an alternative to the Go/No-go task [11]. In an Active

449 choice task, the animals must perform an action directed towards both the positive and

450 negative stimuli, instead of simply displaying an absence of response to the negative

451 stimuli.

452

453

\section{Conclusion}

454

In conclusion, we did not find evidence of a positive judgement bias after goats

had been groomed. To exclude that these results were due to the inefficacy of the grooming to induce an emotional change, we performed a second experiment in which physiological parameters were recorded. We found an increase in heart rate when goats were groomed, suggesting that they were sensitive to the treatment. Thus, the grooming potentially induced an emotional change but this was not detected during the judgement bias test. The performance in the judgment bias test was influenced by the age of the animals. Our findings demonstrate the importance of combining behavioural, physiological and cognitive factors to assess the emotional states experienced by animals. In addition, taking into account individual characteristics of the animals (e.g. age, sex and personality [34]) and clarifying which emotional states are identifiable by a 
465 judgment bias paradigm could increase the effectiveness of cognitive bias paradigms to 466 assess emotional valence [10].

\section{7}

468 Acknowledgements

469

We thank Eric Romero Gonzalez for helping with data collection, Marie-Sophie

470 Single for video analysis, and Caroline Spence and the editor and reviewers for their

471 helpful comments. We thank Bob Hitch, Gower McCarthy, Samantha Taylor and all the

472 volunteers at Buttercups Sanctuary for Goats (http://www.buttercups.org.uk) for their

473 excellent help and free access to the animals. 


\section{References}

475 [1] J. Panksepp, Affective consciousness: core emotional feelings in animals and humans. Conscious. Cogn. 14 (2005) 30-80.

477 [2] J. Burgdorf, J. Panksepp, The neurobiology of positive emotions. Neurosci. Biobehav. Rev. 30 (2006) 173-87.

[3] M. Mendl, O.H.P. Burman, R.M.A. Parker, E.S. Paul, Cognitive bias as an indicator of animal emotion and welfare: emerging evidence and underlying mechanisms. Appl. Anim. Behav. Sci. 118 (2009) 161-181.

[4] M. Mendl, O.H.P. Burman, E.S. Paul, An integrative and functional framework for the study of animal emotion and mood. Proc. R. Soc. B. 277 (2010) 2895-904.

[5] E.F. Briefer, F. Tettamanti, A.G. McElligott, Emotions in goats: mapping physiological, behavioural and vocal profiles. Anim. Behav. 99 (2015) 131-143.

[6] L. Désiré, A. Boissy, I. Veissier, Emotions in farm animals: a new approach to animal welfare in applied ethology. Behav. Proc. 60 (2002) 165-180.

A. Boissy, G. Manteuffel, M.B. Jensen, R.O. Moe, B. Spruijt, L.J. Keeling, C.

Winckler, B. Forkman, I. Dimitrov, J. Langbein, M. Bakken, I. Veissier, Assessment of positive emotions in animals to improve their welfare. Physiol. Behav. 92 (2007) 375-97.

492

[8] T.E. Nygren, A.M. Isen, P.J. Taylor, J. Dulin, The influence of positive affect on the decision rule in risk situations: focus on outcome (and especially avoidance of loss) rather than probability. Organ. Behav. Hum. Decis. Process. 66 (1996) 5972.

[9] L. Greiveldinger, I. Veissier, A. Boissy, The ability of lambs to form expectations 
497

498

499

500

501

502

503

504

505

506

507

508

509

510

511

512

513

514

515

516

517

518

519

and the emotional consequences of a discrepancy from their expectations.

Psychoneuroendocrinology. 36 (2011) 806-15.

[10] L. Baciadonna, A. McElligott, The use of judgement bias to assess welfare in farm livestock. Anim. Welf. 24 (2015) 81-91.

[11] S. Roelofs, H. Boleij, R. Nordquist, F.J. Van Der Staay, Making decisions under ambiguity: judgment bias tasks for assessing emotional state in animals. Front. Behav. Neurosci. 10 (2016) 1-16.

[12] A. Destrez, V. Deiss, C. Belzung, C. Lee, A. Boissy, Does reduction of fearfulness tend to reduce pessimistic-like judgment in lambs? Appl. Anim. Behav. Sci. 139 (2012) 233-241.

[13] R.R. Daros, J.H.C. Costa, M.A.G. von Keyserlingk, M.J. Hötzel, D.M. Weary, Separation from the dam causes negative judgement bias in dairy calves. PLOS ONE 9 (2014) e98429.

[14] E. Verbeek, D. Ferguson, P. Quinquet de Monjour, C. Lee, Generating positive affective states in sheep: the influence of food rewards and opioid administration. Appl. Anim. Behav. Sci. 154 (2014) 39-47.

[15] S.M. Matheson, L. Asher, M. Bateson, Larger, enriched cages are associated with "optimistic" response biases in captive European starlings (Sturnus vulgaris). Appl. Anim. Behav. Sci. 109 (2008) 374-383.

[16] A. Wichman, L.J. Keeling, B. Forkman, Cognitive bias and anticipatory behaviour of laying hens housed in basic and enriched pens. Appl. Anim. Behav. Sci. 140 (2012) 62-69.

[17] E.F. Briefer, A.G. McElligott, Rescued goats at a sanctuary display positive mood 
after former neglect. Appl. Anim. Behav. Sci. 146 (2013) 45-55.

521 [18] R. Rygula, H. Pluta, P. Popik, Laughing rats are optimistic. PLOS ONE 7 (2012)

522 e51959.

523 [19] M.C. McGuire, K.L. Williams, L.L.M. Welling, J. Vonk, Cognitive bias in rats is not influenced by oxytocin. Front. Psychol. 6 (2015) 1306.

525

526

527

528

529

530

531

532

533

534

535

536

537

538

539

540

541

542

[20] E. Verbeek, D. Ferguson, C. Lee, Are hungry sheep more pessimistic? The effects of food restriction on cognitive bias and the involvement of ghrelin in its regulation. Physiol. Behav. 123 (2014) 67-75.

[21] C. Tallet, I. Veissier, X. Boivin, Human contact and feeding as rewards for the lamb's affinity to their stockperson. Appl. Anim. Behav. Sci. 94 (2005) 59-73.

[22] S. Waiblinger, X. Boivin, V. Pedersen, M.-V. Tosi, A.M. Janczak, E.K. Visser,R.B. Jones, Assessing the human-animal relationship in farmed species: a critical review. Appl. Anim. Behav. Sci. 101 (2006) 185-242.

[23] S. Lürzel, I. Windschnurer, A. Futschik, S. Waiblinger, Gentle interactions decrease the fear of humans in dairy heifers independently of early experience of stroking. Appl. Anim. Behav. Sci. (2016) 6-9.

[24] M. Coulon, R. Nowak, J. Peyrat, H. Chandèze, A. Boissy, X. Boivin, Do lambs perceive regular human stroking as pleasant? Behavior and heart rate variability analyses. PLOS ONE 10 (2015) e0118617.

[25] M.A. Zeder, The initial domestication of goats (Capra hircus) in the Zagros Mountains 10,000 years ago. Science 287 (2000) 2254-2257.

[26] C. Nawroth, J.M. Brett, A.G. McElligott, Goats display audience-dependent human-directed gazing behaviour in a problem-solving task. Bilogy Lett. 12 (2016) 
20160283.

544 [27] C. Schmied, S. Waiblinger, T. Scharl, F. Leisch, X. Boivin, Stroking of different body regions by a human: effects on behaviour and heart rate of dairy cows. Appl. Anim. Behav. Sci. 109 (2008) 25-38.

[28] H.S. Proctor, G. Carder, Can ear postures reliably measure the positive emotional state of cows? Appl. Anim. Behav. Sci. 161 (2014) 20-27.

[29] A. Destrez, V. Deiss, C. Leterrier, L. Calandreau, A. Boissy, Repeated exposure to positive events induces optimistic-like judgment and enhances fearfulness in chronically stressed sheep. Appl. Anim. Behav. Sci. 154 (2014) 30-38.

L. Baciadonna, A.G. McElligott, E.F. Briefer, Goats favour personal over social information in an experimental foraging task. PeerJ. 1 (2013) e172.

$$
\text { C. Schmied, X. Boivin, S. Waiblinger, Stroking different body regions of dairy }
$$
cows: effects on avoidance and approach behavior toward humans. J. Dairy Sci. 91 (2008) 596-605.

[32] F.A.M. Tuyttens, S. de Graaf, J.L.T. Heerkens, L. Jacobs, E. Nalon, S. Ott, L. Stadig, E. Van Laer, B. Ampe, Observer bias in animal behaviour research: Can we believe what we score, if we score what we believe? Anim. Behav. 90 (2014) 273-280.

[33] E. von Borell, J. Langbein, G. Després, S. Hansen, C. Leterrier, J. MarchantForde, R. Marchant-Forde, M. Minero, E. Mohr, A. Prunier, D. Valance, I. Veissier, Heart rate variability as a measure of autonomic regulation of cardiac activity for assessing stress and welfare in farm animals - a review. Physiol. Behav. 92 (2007) 293-316. 
566 [34] E.F. Briefer, J.A. Oxley, A.G. McElligott, Autonomic nervous system reactivity in a

567 free-ranging mammal: Effects of dominance rank and personality. Anim. Behav.

$568 \quad 110(2015) 121-132$.

569 [35] S.J.C. Pinheiro, Mixed-Effects Models in S and S-PLUS. S Jose C. Pinheiro, Springer-Verlag, NewYork, 2000.

571

572

573

574

575

576

577

578

579

580

581

582

583

584

585

586

587

588

[36] R. Development Core Team. (2013). R Foundation for Statistical Computing. Vienna: Austria. Retrieved from http://www.R-project.org (accessed March 16, 2016).

[37] Association for the Study of Animal Behaviour, Guidelines for the treatment of animals in behavioural research and teaching. Anim. Behav. 87 (2014) I-IX.

[38] S. Lürzel, C. Münsch, I. Windschnurer, A. Futschik, R. Palme, S. Waiblinger, The influence of gentle interactions on avoidance distance towards humans, weight gain and physiological parameters in group-housed dairy calves. Appl. Anim. Behav. Sci. 172 (2015) 9-16.

[39] N. Reefmann, B. Wechsler, L. Gygax, Behavioural and physiological assessment of positive and negative emotion in sheep. Anim. Behav. 78 (2009) 651-659.

[40] M. Zebunke, B. Puppe, J. Langbein, Effects of cognitive enrichment on behavioural and physiological reactions of pigs. Physiol. Behav. 118 (2013) 7079.

[41] N. Reefmann, F.B. Kaszàs, B. Wechsler, L. Gygax, Physiological expression of emotional reactions in sheep. Physiol. Behav. 98 (2009) 235-241.

[42] S. Waiblinger, C. Menke, J. Korff, A. Bucher, Previous handling and gentle interactions affect behaviour and heart rate of dairy cows during a veterinary 
procedure. Appl. Anim. Behav. Sci. 85 (2004) 31-42.

590

591

592

593

594

595

596

597

598

599

600

601

602

603

604

605

606

607

608

609

610

611

[43] M. Zupan, J. Buskas, J. Altimiras, L.J. Keeling, Assessing positive emotional states in dogs using heart rate and heart rate variability. Physiol. Behav. 155 (2015) 102-111.

[44] T. Travain, E.S. Colombo, L.C. Grandi, E.U.L. Heinzl, A. Pelosi, E. Prato-Previde, P. Valsecchi, How good is this food? A study on dogs' emotional responses to a potentially pleasant event using infrared thermography. Physiol. Behav. 159 (2016) 80-87.

[45] G. Schino, R. Massimei, M. Pinzaglia, E. Addessi, Grooming, social rank and "optimism" in tufted capuchin monkeys: a study of judgement bias. Anim. Behav. 119 (2016) 11-16.

[46] DEFRA, Department for Environment, Animal Welfare Act 2006, (2006). http://www.webarchive.nationalarchives.gov.uk (accessed February 18, 2015).

[47] G. Schino, Reconciliation in domestic goats. Behaviour. 135 (1998) 343-356.

[48] J. Weafer, H. de Wit, Sex differences in impulsive action and impulsive choice. Addict. Behav. 39 (2014) 1573-9.

[49] M.E. Andrzejewski, T.L. Schochet, E.C. Feit, R. Harris, B.L. McKee, A.E. Kelley, A comparison of adult and adolescent rat behavior in operant learning, extinction, and behavioral inhibition paradigms. Behav. Neurosci. 125 (2011) 93-105.

[50] C.L. Burton, P.J. Fletcher, Age and sex differences in impulsive action in rats: the role of dopamine and glutamate. Behav. Brain Res. 230 (2012) 21-33.

[51] G. Laviola, W. Adriani, M. Rea, L. Aloe, E. Alleva, Social withdrawal, neophobia, and stereotyped behavior in developing rats exposed to neonatal asphyxia. 
$612 \quad$ Psychopharmacology 175 (2004) 196-205.

613 [52] T.L. Doremus, E.I. Varlinskaya, L.P. Spear, Age-related differences in elevated

614 plus maze behavior between adolescent and adult rats. Ann. N. Y. Acad. Sci.

$615 \quad 1021(2004) 427-30$. 
616 Table 1 Characteristics of goats tested in the judgement bias experiment: ID, breed,

617 age, sex, treatment and rewarded side.

\begin{tabular}{llllll}
\hline ID & Breed & Age & Sex & Treatment & Rewarded Side \\
\hline 1 & Mixed breed & 7 & Male & Control & Right \\
2 & British Toggenburg & 11 & Male & Grooming & Left \\
3 & British Toggenburg & 10 & Male & Grooming & Left \\
4 & Golden Guernsey & 9 & Male & Grooming & Right \\
5 & Pygmy Goat & 6 & Male & Control & Left \\
6 & British Toggenburg & 3 & Male & Grooming & Right \\
7 & Mixed breed & 14 & Male & Control & Left \\
8 & Mixed breed & 9 & Male & Control & Right \\
9 & Mixed breed & 9 & Male & Control & Left \\
10 & British Alpine & 8 & Female & Control & Right \\
11 & British Saanen & 10 & Female & Grooming & Right \\
12 & British Toggenburg & 10 & Female & Grooming & Left \\
13 & British Alpine & 10 & Female & Grooming & Right \\
14 & British Saanen & 4 & Female & Control & Left \\
15 & British Saanen & 4 & Female & Control & Right \\
16 & British Toggenburg & 2 & Female & Grooming & Left \\
17 & British Toggenburg & 3 & Female & Grooming & Left \\
18 & Anglo Nubian & 8 & Female & Control & Right \\
19 & Boer & 1 & Female & Control & Left \\
\hline
\end{tabular}




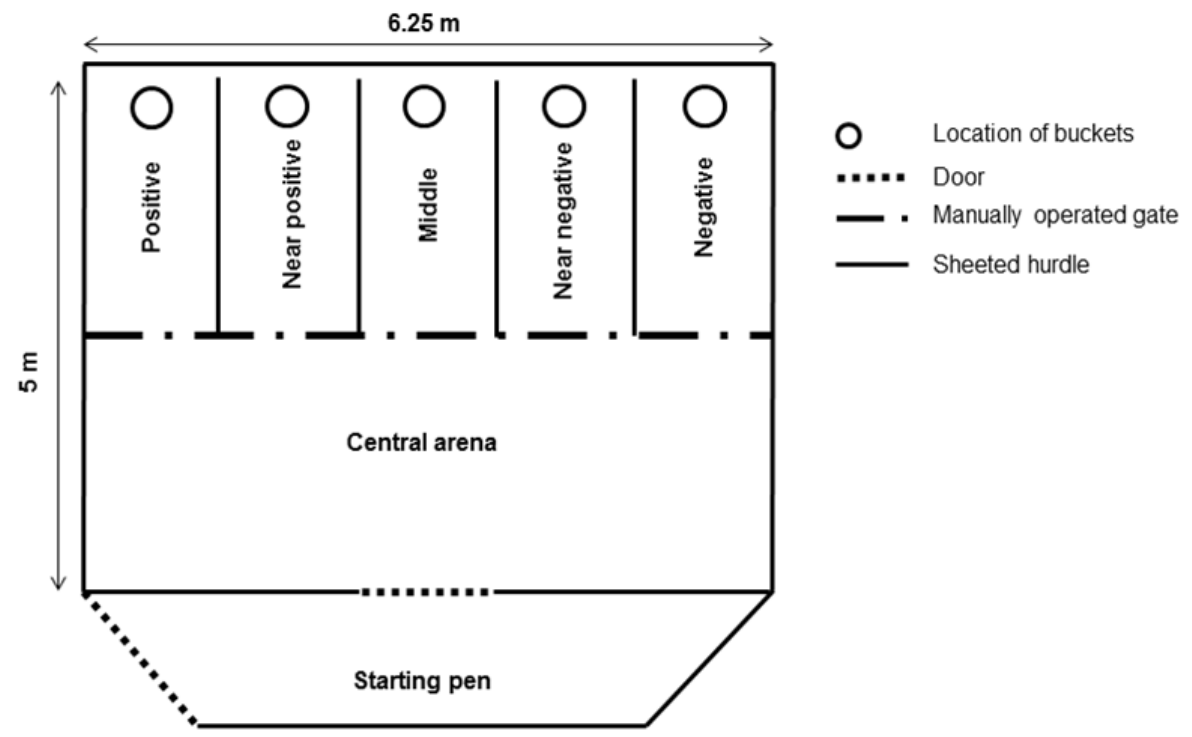

620 Figure 1 Experimental apparatus. Position of the positive corridor (right or left

621 depending on the goats), the negative corridor (opposite direction), the three ambiguous

622 corridors, the central arena and the start pen. The latency to reach the locations was

623 measured (distance from the start pen to the beginning of each corridor). 


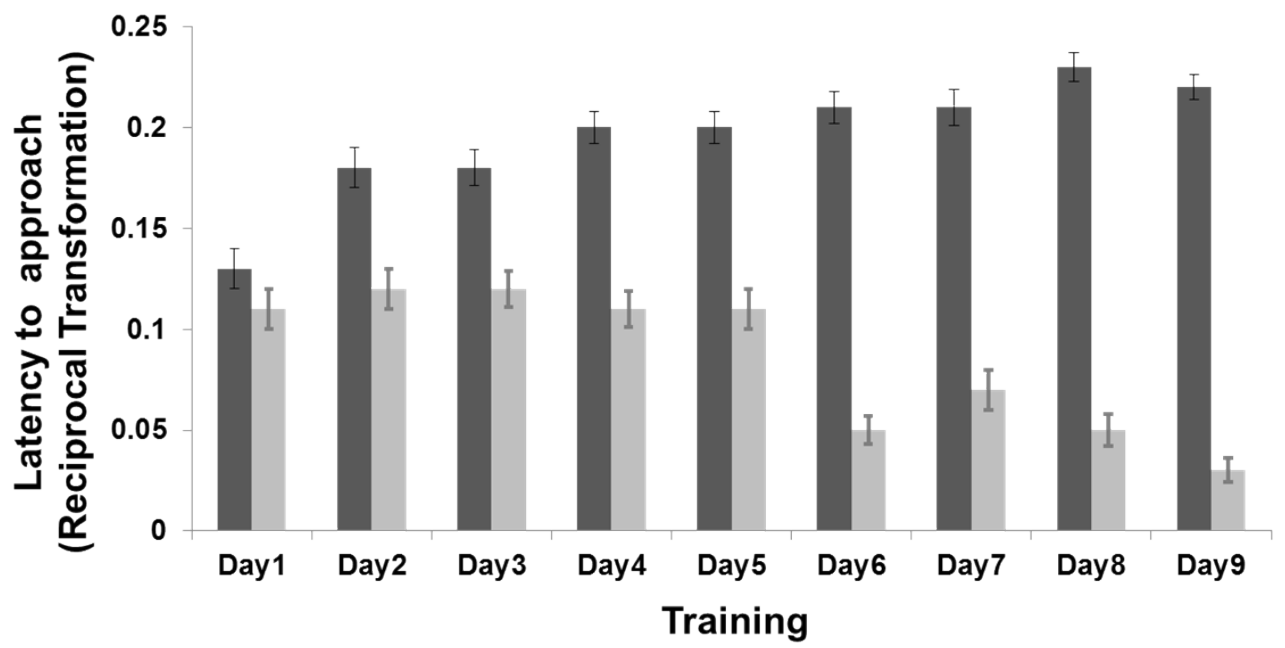

625

626 Figure 2 Results of the training phase. Latency (Mean \pm SE) to reach the positive

627 location (dark grey bar) and the negative location (light grey bar) during the nine days of

628 training. The latency time was transformed $(1 / X i)$, and therefore higher latency times

629 indicate faster approaches and vice versa. There was an interaction effect between

630 training day and locations (LMM: $p<0.001)$. 


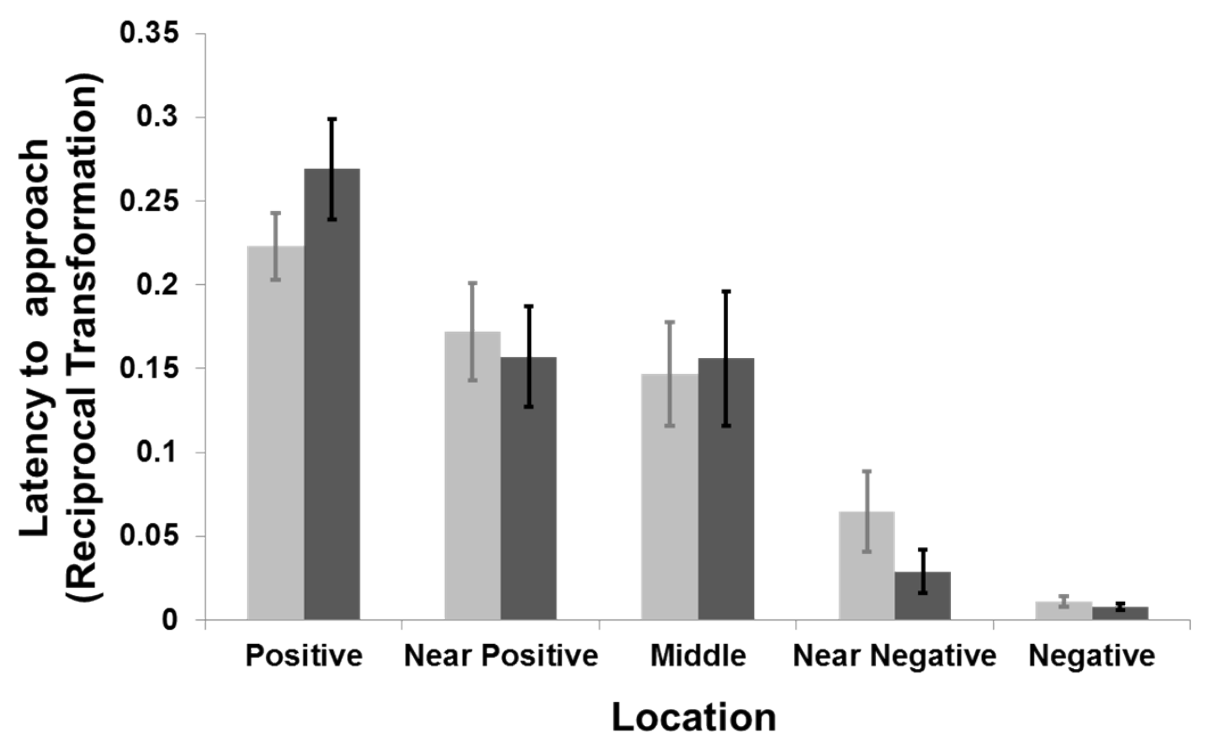

632

633 Figure 3 Results of the judgement bias experiment. Latency (Mean \pm SE) to reach the

634 five locations during the two days of test, for the groomed group (dark grey bar), and the 635 control group (light grey bar). The latency time was transformed (1/Xi), and therefore

636 higher latency times indicate faster approaches and vice versa.There was a general

637 effect of location (LMM: $p<0.001$ ), but no interaction between locations and treatment $638($ LMM $p>0.39)$. 


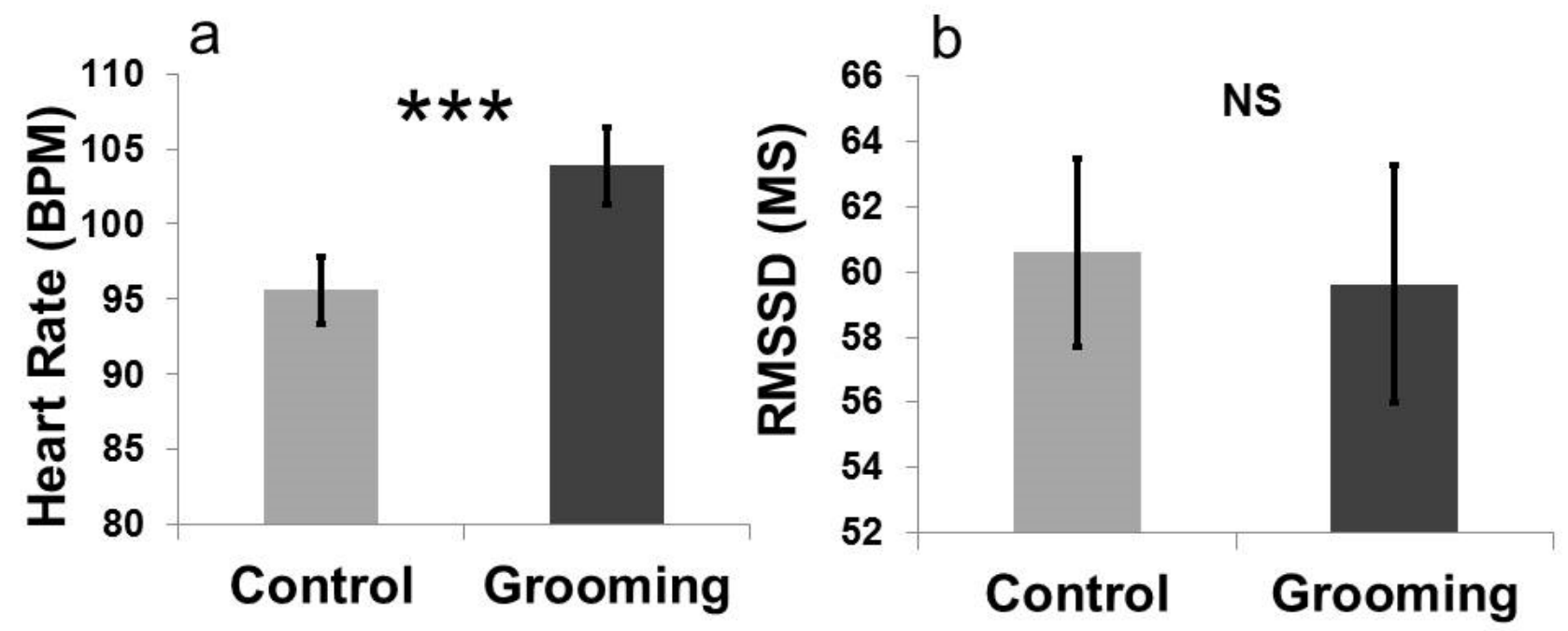

Figure 4. (a) Heart rate (measured on the same animals) increased when goats were

642 groomed compared to when the same animals were kept close to the experimenter

643 without being groomed. (b) Heart rate variability (RMSSD) was not significantly different

644 in the grooming compared to the control treatment. 